Louisiana State University

LSU Digital Commons

$5-12-2009$

\title{
Contemporary proton therapy systems adequately protect patients from exposure to stray radiation
}

\author{
Wayne D. Newhauser \\ University of Texas Health Science Center at Houston \\ Jonas D. Fontenot \\ University of Texas Health Science Center at Houston \\ Phillip J. Taddei \\ University of Texas Health Science Center at Houston \\ Dragan Mirkovic \\ University of Texas Health Science Center at Houston \\ Annelise Giebeler \\ University of Texas Health Science Center at Houston
}

See next page for additional authors

Follow this and additional works at: https://digitalcommons.Isu.edu/physics_astronomy_pubs

\section{Recommended Citation}

Newhauser, W., Fontenot, J., Taddei, P., Mirkovic, D., Giebeler, A., Zhang, R., Mahajan, A., Kornguth, D., Stovall, M., Yepes, P., Woo, S., \& Mohan, R. (2009). Contemporary proton therapy systems adequately protect patients from exposure to stray radiation. AIP Conference Proceedings, 1099, 450-455. https://doi.org/10.1063/1.3120071

This Conference Proceeding is brought to you for free and open access by the Department of Physics \& Astronomy at LSU Digital Commons. It has been accepted for inclusion in Faculty Publications by an authorized administrator of LSU Digital Commons. For more information, please contact ir@lsu.edu. 


\section{Authors}

Wayne D. Newhauser, Jonas D. Fontenot, Phillip J. Taddei, Dragan Mirkovic, Annelise Giebeler, Rui Zhang, Anita Mahajan, David Kornguth, Marilyn Stovall, Pablo Yepes, Shiao Woo, and Radhe Mohan 


\title{
Contemporary Proton Therapy Systems Adequately Protect Patients from Exposure to Stray Radiation
}

\author{
Wayne D. Newhauser ${ }^{1},{ }^{*}$, Jonas D. Fontenot ${ }^{1}$, Phillip J. Taddei ${ }^{1}$, Dragan Mirkovic ${ }^{1}$, Annelise \\ Giebeler ${ }^{1}$, Rui Zhang ${ }^{1}$, Anita Mahajan ${ }^{1}$, David Kornguth ${ }^{1}$, Marilyn Stovall ${ }^{1}$, Pablo Yepes ${ }^{2}$, \\ Shiao Woo ${ }^{1}$, and Radhe Mohan ${ }^{1}$ \\ ${ }^{1}$ Department of Radiation Physics, The University of Texas M. D. Anderson Cancer Center, 1515 \\ Holcombe Blvd, Unit 94, Houston, TX 77030, USA \\ ${ }^{2}$ Department of Physics \& Astronomy, MS 61, Rice University, 6100 Main St, Houston, TX 77005, \\ United States
}

\begin{abstract}
Proton beam therapy has provided safe and effective treatments for a variety of adult cancers. In recent years, there has been increasing interest in utilizing proton therapy for pediatric cancers because it allows better sparing of healthy tissues. Minimizing exposures of normal tissues is especially important in children because they are highly susceptible to consequential late effects, including the development of a radiogenic second cancer, which may occur years or even decades after treatment of the first cancer. While the dosimetric advantage of therapeutic proton beams is well understood, relatively little attention has been paid to the whole-body exposure to stray neutron radiation that is inherent in proton therapy. In this report, we review the physical processes that lead to neutron exposures, discuss the potential for mitigating these exposures using advanced proton beam delivery systems, and present a comparative analysis of predicted second cancer incidence following various external beam therapies. In addition, we discuss uncertainties in the relative biological effectiveness of neutrons for carcinogenesis and the impact that these uncertainties have on second-cancer risk predictions for survivors of adult and childhood cancer who receive proton therapy.
\end{abstract}

\section{Keywords}

proton therapy; intensity-modulated radiation therapy; radiogenic second cancer

\section{INTRODUCTION}

The efficacy of cancer radiotherapy has improved dramatically over the last several decades and patients are now surviving longer and in greater numbers. ${ }^{1}$ With this progress, increasing attention is being paid to improving patients' quality of life. Much of the contemporary research in conventional radiation oncology is devoted to reducing radiation toxicity in healthy tissues, e.g., through more accurate targeting of the radiation beam on the tumor. Another area of investigation is the use of high-energy proton beams in place of photon beams because proton beams inherently allow superior sparing of surrounding healthy tissues, as demonstrated in several treatment planning studies. ${ }^{2,3}$ For example, by adjusting the energy of the proton beam 
so that it stops just beyond the tumor, the normal tissues downstream are spared the so called "exit dose" associated with exponentially-attenuated photon beams. This sparing of normal tissue from the therapeutic beam theoretically leads to a reduction in the severity or incidence of side effects.

The side effects from radiotherapy are commonly classified as acute or late. Acute effects, which may appear minutes to weeks after treatment, are reasonably well understood for photon and proton therapy. Typically, acute effects are relatively minor, such as skin irritation and hair loss, but infrequently they may be more severe and, in rare cases, fatal. Late effects, which may appear years or decades after treatment are less understood, particularly for proton therapy. A late effect of great concern is the development of a radiogenic second cancer in a patient who survives the first cancer; this concern in heightened in treatment of children because of their greater sensitivity to radiation carcinogenesis. ${ }^{4}$ Our present knowledge of the risks associated with stray radiation emanating from the treatment unit versus scatter radiation emanating from the region of the tumor is incomplete. The literature contains only limited measurements ${ }^{5,6}$ and simulations ${ }^{7-10}$ of the stray and leakage radiation levels near operational high-capacity proton therapy machines. Furthermore, there is a lack of sufficient epidemiological data to support a physically complete prediction and direct comparison of predicted risk of second cancer associated with proton versus photon therapy and passivelyscattered versus magnetically-scanned proton beam therapy. Previous treatment planning studies suggested that proton therapy is superior to photon therapy in terms of risk ${ }^{2,11}$ but they did not take into account risks associated with stray neutron radiation. Neutron radiation is particularly effective at causing cancer, and consequently, the true risks associated with proton therapy have become a matter of controversy. ${ }^{12}$

The objective of this work was to compare the risk of radiogenic cancer following proton versus photon therapy. In particular, we calculated the ratios of relative risk for the development of a second cancer associated with passively-scattered proton therapy, intensity-modulated proton therapy (IMPT) delivered with scanned beams, and conventional intensity-modulated photon radiation therapy (IMRT). Risk analyses were carried out for patients diagnosed with brain and prostate cancer. Radiation doses were calculated using analytical dose algorithms or Monte Carlo models. Risks were calculated using the organ doses and coefficients of risk per unit dose from the literature.

\section{METHODS AND MATERIALS}

We considered two important types of cancer with particular relevance to the comparison of second cancer risks. Prostate cancer is prevalent and potentially deadly; there are more than 186,000 new cases diagnosed and more than 28,000 deaths from this disease per year in the United States. Furthermore, prostate cancer comprises an important segment of the total caseload of proton therapy, e.g., approximately $60 \%$ of patients receiving proton therapy in the United States are being treated for prostate cancer. Furthermore, the treatment of prostate cancer provides a severe and interesting test of proton therapy's ability to limit the risk of second cancer, for two reasons. First, the prostate is deeply seated in the pelvis (at approximately $24 \mathrm{~cm}$ water equivalent depth from the skin to the center of the prostate) and located between two radiosensitive organs (the bladder and rectum). Second, the prostate requires a higher beam energy than most anatomical sites, typically about $225 \mathrm{MeV}$, and the production of stray neutron radiation increases supralinearly with proton beam energy. ${ }^{9}$

The second type of treatment considered in this study was supratentorial primitive neuroectodermal tumor, a cancer of the central nervous system that occurs in children and young adults. The standard treatment includes irradiation of the whole brain and spine (craniospinal irradiation, or CSI). Although childhood cancer is comparatively rare 
(approximately 12,400 new cases per year arise in people less than 20 years old in the United States), it is important to consider pediatric treatments for several reasons. As noted above, children are more sensitive to radiation carcinogenesis than adults and, by virtue of their youth and good prognoses, most will survive longer than the latency periods for the development of second cancers. Furthermore, the spinal treatment fields comprise a severe test of passivelyscattered proton therapy in terms of neutron production in the collimator. Specifically, the long and narrow spinal fields cause a large fraction of the protons to be intercepted in the final collimator, which increases neutron production.

We calculated the therapeutic radiation doses to organs and tissues with a commercial treatment planning system (TPS) (Eclipse, Varian Medical Systems, Palo Alto, CA). However, for the CSI case, the therapeutic absorbed doses used in the risk analysis were taken from Miralbell et $a$. $^{2}$ in order to preserve consistency with that study. The stray neutron radiation doses were calculated with an in-house Monte Carlo (MC) dose engine13 (the results of which are shown in Figures 1 and 2) based on the general purpose MCNPX code.14 The accuracy of the TPS13 and MC system ${ }^{14}$ was previously established. Absorbed doses to organs and tissues were calculated separately for neutrons emanating from the treatment apparatus (external neutrons) and those generated inside the patient (internal neutrons). Absorbed dose was converted to equivalent dose using mean neutron radiation weighting factors $\left(w_{R}\right)$ that were calculated based on calculated neutron spectral fluences ${ }^{16}$ and from recommended values of $w_{\mathrm{R}}$ versus neutron energy, as provided in International Commission on Radiation Protection (ICRP) Publication 92. ${ }^{17}$ The ICRP's "best estimate" of the maximum $w_{\mathrm{R}}$ value at any neutron energy was 20 . The mean $w_{\mathrm{R}}$ values for the neutron energy distributions in the CSI patient were 8 for passively-scattered beams and 9 for scanned beams. In addition, the recommended $w_{\mathrm{R}}$ values were scaled by $0.5,2$ and 5 for both the prostate and CSI cases in order to test the sensitivity of the risk prediction on possible systematic biases. The treatment techniques were described previously, ${ }^{18}$ including clinical parameters such as beam angles, energies, and collimation.

We defined the quantity ratio of excess relative risk $(R R R)$ to evaluate the risk from proton therapy relative to IMRT. The $R R R$ is defined as

$$
R R R=E R R_{\text {proton }} / E R R_{\mathrm{IMRT}}
$$

where $E R R_{\text {proton }}$ and $E R R_{\mathrm{IMRT}}$ are the patient's excess relative risk $(E R R)$ values for developing a radiation-induced cancer anywhere in the body after undergoing proton therapy and IMRT, respectively. The quantity $E R R$ is an epidemiologic quantity used to describe the instantaneous rate (number per year) of excess incidence of fatal and nonfatal solid cancers in an exposed population relative to an unexposed population. Values of ERR for each modality (proton therapy and IMRT) were calculated according to

$$
E R R_{\text {modality }}=\left(\sum_{\mathrm{T}} E R R_{\mathrm{T}}\right)_{\text {modality }}
$$

where $E R R_{\mathrm{T}}$ is the excess relative risk of developing a radiation-induced cancer in organ or tissue T. The value of $E R R_{\mathrm{T}}$ was calculated using

$$
E R R_{\mathrm{T}}=H_{\mathrm{T}} \times\left(\frac{E R R}{H}\right)_{\mathrm{T}}
$$


where $H_{\mathrm{T}}$ is equivalent dose to the organ or tissue and $\left(\frac{E R R}{H}\right)$ is an organ-specific risk coefficient. In this work, organ-specific risk coefficients were taken from the National Academy of Science ${ }^{19}$ for the prostate treatment and from ICRP Publication $60^{20}$ for the CSI. For the prostate case, adjustments were made for age at exposure, and attained age. ${ }^{21}$ Values of $H_{\mathrm{T}}$ were calculated as the product of the absorbed dose to a tissue or organ, $D_{\mathrm{T}}$, and the mean radiation weighting factor, $w_{\mathrm{R}}$, as described above. For the CSI case, adjustments were made for attained age. ${ }^{16}$ The prescribed doses to the target volumes were 69.1 Gy and 36 Gy for the prostate and CSI cases, respectively.

\section{RESULTS}

The predicted $R R R$ value for the prostate cancer patient considered was 0.66 , indicating that the risk following passively-scattered proton therapy is markedly lower than that following IMRT. To place this result in the context of absolute risk, we note that clinical outcome studies suggest that the ERR at 10 years after external-beam photon radiotherapy of the prostate is less than $2 \%$ (see Fontenot et al21). The passively-scattered proton treatment resulted in an effective dose of just $415 \mathrm{mSv}$ due to stray radiation. Again, to help place this in context, a recent simulation study revealed that with additional shielding and collimation in the treatment unit, a passively-scattered treatment can be delivered with stray radiation exposures just $109 \mathrm{mSv}$ larger than those from an idealized scanned-beam proton treatment.22 This corresponds to approximately $0.5 \%$ difference in the $E R R$ values from passively-scattered and scanned proton treatments.

Figure 3 plots the proportions of equivalent dose in various organs and tissues contributed by internal and external neutrons and photons for the prostate case.

Figure 4 plots the dependence of $R R R$ on the scaling factor applied to the $w_{\mathrm{R}}$ values for neutrons. The $E R R$ values for passively-scattered proton therapy and IMRT were equal when the scaling factor for the $w_{\mathrm{R}}$ value for neutrons was increased by a factor of 4.8 .

For the CSI treatment with passively-scattered protons, our predicted $R R R$ value was 0.16 , indicating that the risk was markedly lower after proton therapy than after IMRT. For context, the predicted lifetime ERR was $31 \%$ following IMRT versus $5.1 \%$ following the passivelyscattered proton treatment. The passively-scattered proton treatment produced an effective dose of only $188 \mathrm{mSv}$ from stray neutron radiation. The idealized scanned-beam proton treatment yielded $89 \mathrm{mSv}$, a reduction of only $99 \mathrm{mSv}$ of stray radiation compared to the passively-scattered proton treatment, corresponding to a reduction of just $0.7 \%$ in lifetime $E R R$. The $R R R$ risk predictions for the CSI case are summarized in Table 1. Interestingly, the $R R R$ values indicate that proton therapy carries lower predicted risks than IMRT for any value of the scaling factor (applied to the neutron $w_{\mathrm{R}}$ values) less than 19.

\section{DISCUSSION}

In this study we compared the risk of developing a second cancer following proton therapy versus IMRT for two patients. In the case of the patient with prostate cancer, the risk following passively-scattered proton therapy was approximately two thirds of the risk following IMRT with photon beams. However, in the improbable event that the true maximum radiation weighting factor for the stray neutrons for patients with prostate cancer was five times higher than the value recommended by the ICRP, the risk following proton therapy would be higher. For the CSI treatment, the risk following proton therapy was also lower-about one sixth the risk following IMRT. For this patient, the risk following proton therapy would be higher only 
if the true maximum $w_{R}$ value for neutrons were 19 times higher than the value we calculated using the recommendations of the ICRP.

These results suggest that the risk of developing a second cancer following proton therapy is less than the risk following IMRT for both cases considered. Furthermore, our results indicate that only small additional benefit may be gained by the more demanding method of spot scanning, as evidenced by our finding that the difference in effective dose from passivelyscattered versus scanned proton treatments was only about $100 \mathrm{mSv}$. This additional effective dose corresponds to an increase of less than $1 \%$ in the lifetime risk of second cancer. For context, the CSI patient's predicted lifetime risk of developing a second cancer was $31 \%$ following IMRT, 5.1\% following passively-scattered proton therapy, and $4.4 \%$ following IMPT. These increases in risk include all cancers (fatal and non-fatal) and take into account exposures to both therapeutic and stray radiation.

The risk results may be summarized as follows. The risk of second cancer following IMRT $(31 \%)$ is clinically significant. By using passively-scattered proton therapy instead of IMRT, the predicted risk drops dramatically to a level that is small but not clinically negligible (5.1\%). By using scanned proton beams in place of passively-scattered proton beams for CSI, the predicted risk drops farther but by less than $1 \%$. Qualitatively, the risk analysis for the prostate treatment was similar to that for the CSI patient, but the predicted risks and risk differentials were much smaller. The findings for these patients suggest that proton therapy carries a lower risk of second cancer and that the difference in $R R R$ between passively-scattered and scannedbeam proton treatments are small and probably clinically negligible.

A noteworthy strength of this study is that it used detailed and clinically realistic models of the treatment, including the beam delivery apparatus, patient-specific beam-modifying devices, and patient. In addition, the radiation transport calculations and risk calculations included the therapeutic and stray radiation components. This has potentially important implications in the interpretation of exposure and risk data from several previous studies. Specifically, by comparing the results from several recent studies, it appears that the scattering and attenuation of radiation inside the patient anatomy can significantly influence the predicted exposure and risk. This was apparent in a series of Monte Carlo studies in which the modeled level of detail in the treatment apparatus and anatomy was progressively increased. ${ }^{7-9,18,21,22}$ Together, the results from these studies suggest that a high level of detail and realism is needed to predict reliable $E R R$ and $R R R$ values for individual patients or for populations of patients, e.g., patients with prostate cancer. At present, the availability of accurate $E R R$ and $R R R$ values is limited to a few case studies and anatomical sites. Additional investigations will be necessary to determine if our present understanding from these case studies holds true for a broad spectrum of pediatric and adult patients. Such data would lead to more accurate cost-benefit analyses of proton therapy versus photon therapy because the costs savings from avoided second cancers could be taken into account.

For those engaged in the research and development of proton beam delivery systems, the implications of this study are more nuanced. In broad terms, our results strengthen the rationale for making proton therapy more widely available. The proliferation of proton therapy facilities is presently constrained because they are more expensive and technically challenging to implement than advanced photon therapy systems. Thus, we believe that future research and development on beam delivery systems should aim to 1) reduce the cost-benefit ratio, 2) decrease the risk of delay of facility startup, and 3) improve the dosimetric performance of proton beam delivery systems.

In our view, research and development is urgently needed for both passive and scanning systems. The vast majority of proton therapy facilities now in operation deliver passively- 
scattered beams, and they will treat tens of thousands of patients in the next decade. The performance of passively-scattered nozzles can and should be dramatically improved ${ }^{22}$ by means of strategies that could be clinically implemented within a few years. At the same time, research should be carried out to improve scanned-beam techniques, such as reducing the proton beam spot size at depth in the patient, improving modulation and control of the instantaneous beam intensity, developing efficient measurement instrumentation for use in the clinic, and solving potential dosimetric problems arising from the interplay of organ motion with the moving proton beam. It will be important to increase the operational efficiency of existing and new facilities that use passive scattering by implementing automation technologies. For example, patient-specific static apertures, which must be manually exchanged for each treatment field, should be replaced with computer-controlled multileaf collimators. ${ }^{23}$

\section{CONCLUSION}

Our investigation corroborates our findings from earlier studies that suggested that the risk of a radiogenic second cancer is lower following proton therapy than following photon therapy. Using scanned proton beams in place of passively-scattered proton beams yields only a small, probably clinically insignificant, further reduction in predicted risk. It must be emphasized that these preliminary findings were based on studies of a few patients and where the predicted risks had uncertainties that were large and difficult to estimate. Definitive findings will require additional case studies and refined estimates of the uncertainties in dose and risk values.

\section{Acknowledgments}

This work was funded in part by a grant from Northern Illinois University through a Department of Defense subcontract (award W81XWH-08-1-0205) and the National Cancer Institute (award 1R01CA131463-01A1).

\section{REFERENCES}

1. Ries, LAG.; Harkins, D.; Krapcho, M., et al. SEER Cancer Statistics Review, 1975-2003. Bethesda, MD: National Cancer Institute; 2006.

2. Miralbell R, Lomax A, Cella L, et al. Int J Radiat Oncol Biol Phys 2002;54(3):824. [PubMed: 12377335]

3. Schneider U, Lomax A, Besserer J, et al. Int J Radiat Oncol Biol Phys 2007;68(3):892. [PubMed: 17459608]

4. Curtis, RE.; Freedman, DM.; Ron, E., et al. New Malignancies Among Cancer Survivors: SEER Cancer Registries, 1973-2000. NIH Publ. No. 05-5302. Bethesda, MD: National Cancer Institute; 2006.

5. Yan X, Titt U, Koehler AM, et al. Nucl Instr Meth 2002;476(1-2):429.

6. Tayama R, Fujita Y, Tadokoro M, et al. Nucl Instr Meth 2006;564(1):532.

7. Zheng Y, Fontenot J, Taddei P, et al. Phys Med Biol 2008;53(1):187. [PubMed: 18182696]

8. Zheng Y, Newhauser WD, Fontenot J, et al. J Nucl Matl 2007;361:289.

9. Zheng Y, Newhauser W, Fontenot J, et al. Phys Med Biol 2007;52(15):4481. [PubMed: 17634645]

10. Moyers MF, Benton ER, Ghebremedhin A, et al. Med Phys 2008;35(1):128. [PubMed: 18293570]

11. Yuh GE, Loredo LN, Yonemoto LT, et al. Cancer J 2004;10(6):386. [PubMed: 15701271]

12. Hall EJ. Technol Cancer Res Treatment 2007;6(4):31.

13. Newhauser W, Fontenot J, Zheng Y, et al. Phys Med Biol 2007;52(15):4569. [PubMed: 17634651]

14. Pelowitz, DB., editor. MCNPXTM User's Manual, Version 2.5.0. Los Alamos, NM: Los Alamos National Laboratory; 2005.

15. Titt U, Sahoo N, Ding X, et al. Phys Med Biol 2008;53(16):4455. [PubMed: 18670050]

16. Newhauser WD, Fontenot JD, Mahajan A, et al. Phys Med Biol. (in press).

17. ICRP, Publication 92. Ann. ICRP. Vol. Vol. 33. New York: Pergamon; 2003. 
18. Fontenot J, Taddei P, Zheng Y, et al. Phys Med Biol 2008;53:1677. [PubMed: 18367796]

19. NRC. Health Risks from Exposure to Low Levels of Ionizing Radation: BEIR VII - Phase 2. Washington D.C.: The National Academies Press; 2006.

20. ICRP, Publication 60. Ann. ICRP. Vol. Vol. 21. New York: Pergamon Press; 1991.

21. Fontenot, JD. Ph. D. thesis, Proton therapy versus intensity modulated x-ray therapy in the treatment of prostate cancer: estimating secondary cancer risks. Houston, TX: Univ. Texas Graduate School of Biomedical Sciences; 2008.

22. Taddei PJ, Fontenot J, Zheng Y, et al. Phys Med Biol 2008;53:2131. [PubMed: 18369278]

23. Bues M, Newhauser WD, Titt U, et al. Radiat Prot Dosim 2005;115(1-4):164.

24. Taddei PJ, Mirkovic D, Fontenot JD, et al. Phys Med Biol. (in press). 

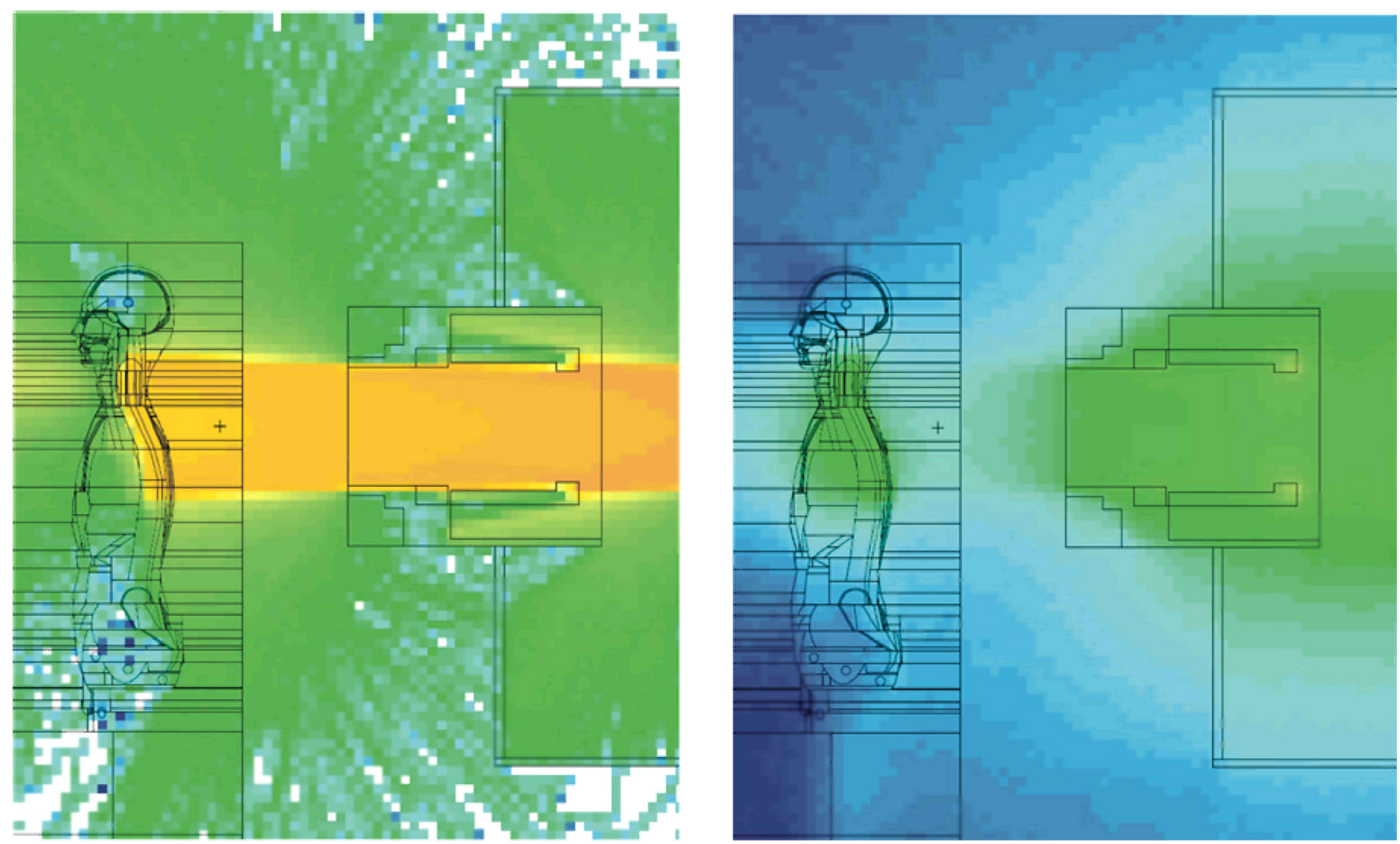

FIGURE 1.

Left: Upper spinal treatment field of a craniospinal treatment with proton beams. The colors denote the logarithm of proton fluence, where the protons were associated with the therapeutic proton beam or with $(\mathrm{n}, \mathrm{xp})$ reactions. Right: Logarithm of neutron fluence associated with the same treatment field. The images are in arbitrary units and are plotted on different scales from one another. The fluence intensity is represented by a color scale that transitions from deep blue (lowest fluence) to deep orange (highest fluence). 


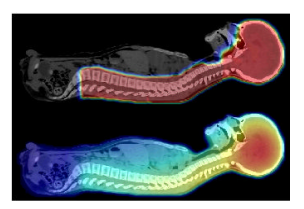

FIGURE 2.

Top: Proton absorbed dose distribution from a four-field proton craniospinal irradiation of a 10-year-old boy with supratentorial primitive neuroectodermal tumor. Bottom: The associated neutron absorbed dose distribution. ${ }^{24}$ Note that for clarity, the images are in arbitrary units and are plotted on different scales from one another. These images are both taken in the mid-sagittal plane. The absorbed dose is represented by a color scale that transitions from dark blue (lowest dose) to red (highest dose). 


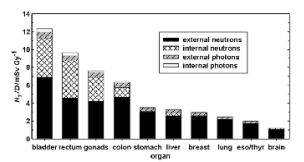

FIGURE 3.

$H_{\mathrm{T}} / D$ values for a passively-scattered proton treatment of the prostate, detailing the contributions from internal and external neutrons and photons. The values for internal radiation represent a scanned-beam treatment that is ideal in the sense that no neutrons emanated from the treatment unit. ${ }^{21}$ 


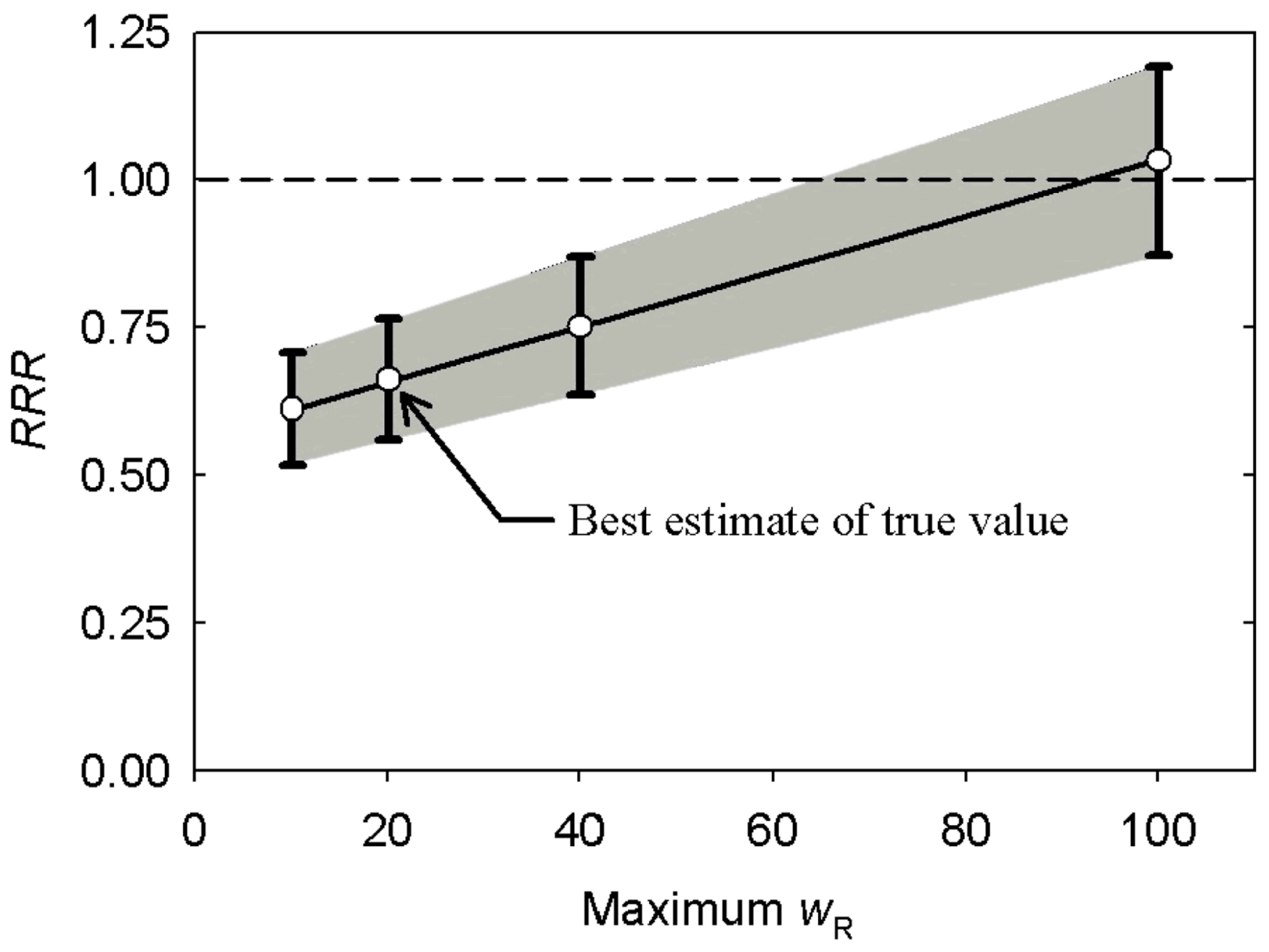

FIGURE 4.

Ratio of relative risk $(R R R)$ versus maximum neutron radiation weighting factor $\left(w_{\mathrm{R}}\right)$, where the "best estimate" of the true maximum value was taken as 20 , following the recommendation of the ICRP. ${ }^{17}$ 


\section{TABLE 1}

Summary of values of relative risk following craniospinal irradiation with intensity-modulated proton therapy (IMPT) and passively-scattered proton therapy (PSPT). These values are based on intensity-modulated radiation therapy (IMRT) absorbed doses and therapeutic proton absorbed doses taken from Miralbell et al. ${ }^{2}$ Stray neutron doses associated with the proton treatment were taken from Newhauser et al. ${ }^{16}$ All values listed take into account risk associated with therapeutic and stray radiation exposures. The values of ratio of relative risk $(R R R)$, which were calculated according to Eq. (1) in the text, are listed here as inverse $R R R$ values for clarity, i.e., to convey the multiples of higher risk following IMRT. The inverse $R R R$ values are tabulated as a function of the multiplicative scaling factor that was applied to the nominal values of radiation weighting factor $\left(w_{R}\right)$ for neutrons.

\begin{tabular}{ccc}
\hline $\begin{array}{c}\text { Scaling } \\
\text { factor for mean } \\
\boldsymbol{w}_{\mathbf{R}} \text { for neutrons }\end{array}$ & $\begin{array}{c}(\boldsymbol{R R R})^{-\mathbf{1}} \\
\text { IMPT }\end{array}$ & $\begin{array}{c}(\boldsymbol{R R R})^{-\mathbf{1}} \\
\text { PSPT }\end{array}$ \\
\hline 1 & 7.1 & 6.1 \\
2 & 6.2 & 4.8 \\
5 & 4.3 & 2.9 \\
19 & 1.8 & 1.0 \\
\hline
\end{tabular}

